\title{
Tracking progressive pathological and functional decline in the rTg4510 mouse model of tauopathy
}

Thomas Blackmore* (D), Soraya Meftah, Tracey Karen Murray, Peter James Craig, Anthony Blockeel, Keith Phillips, Brian Eastwood, Michael J. O'Neill, Hugh Marston, Zeshan Ahmed, Gary Gilmour and Francois Gastambide

\begin{abstract}
Background: The choice and appropriate use of animal models in drug discovery for Alzheimer's disease (AD) is pivotal to successful clinical translation of novel therapeutics, yet true alignment of research is challenging. Current models do not fully recapitulate the human disease, and even exhibit various degrees of regional pathological burden and diverse functional alterations. Given this, relevant pathological and functional endpoints must be determined on a model-by-model basis. The present work explores the rTg4510 mouse model of tauopathy as a case study to define best practices for the selection and validation of cognitive and functional endpoints for the purposes of pre-clinical AD drug discovery.
\end{abstract}

Methods: Male rTg4510 mice were first tested at an advanced age, 12 months, in multiple behavioural assays (step 1). Severe tau pathology and neurodegeneration was associated with profound locomotor hyperactivity and spatial memory deficits. Four of these assays were then selected for longitudinal assessment, from 4 to 12 months, to investigate whether behavioural performance changes as a function of accumulation of tau pathology (step 2). Experimental suppression of tau pathology — via doxycycline administration—was also investigated for its effect on functional performance.

Results: Progressive behavioural changes were detected where locomotor activity and rewarded alternation were found to most closely correlate with tau burden and neurodegeneration. Doxycycline initiated at 4 months led to a $50 \%$ suppression of transgene expression, which was sufficient to prevent subsequent increases in tau pathology and arrest related functional decline.

Conclusions: This two-step approach demonstrates the importance of selecting assays most sensitive to the phenotype of the model. A robust relationship was observed between pathological progression, development of phenotype, and their experimental manipulation-three crucial factors for assessing the translational relevance of future pre-clinical findings.

Keywords: Alzheimer's Disease, Tau, Neurodegeneration, rTg4510, Behaviour, Cognition, Pathology

\footnotetext{
* Correspondence: thomas.blackmore@hertford.ox.ac.uk

Lilly Research Laboratories, Eli Lilly \& Co. Ltd., Windlesham, UK
} 


\section{Background}

The vast societal and economic burden of Alzheimer's disease (AD) represents a growing problem [1]. Despite extensive effort, $\mathrm{AD}$ drug discovery programmes have so far lacked late-stage clinical success [2, 3]. While some of these failures have arisen from incomplete understanding of drug properties or inappropriate study design $[4,5]$, many may also reflect true negative effects. Given the several hundred interventions reported to mitigate pathological and behavioural alterations in AD mouse models, it is reasonable to question the predictive validity of early AD drug discovery [6]. Pre-clinical validation forms an integral part of nearly every drug project, yet justification of disease model as well as the endpoint(s) chosen often appears ad hoc, or simply lacking. In contrast, clinical efficacy of $\mathrm{AD}$ therapies is currently based on pre-defined cognitive test batteries and activities of daily living questionnaires. Therefore, a major consideration for future AD pre-clinical efforts would be to better understand the benefits and limitations of existing behavioural and functional assays, their relationship with disease progression (e.g. [7]), and ultimately with clinical endpoints.

Contemporary research suggests tau rather than amyloid pathology is most closely associated with neurodegenerative processes $[8,9]$ as well as cognitive and functional decline, prompting a shift towards tau-based drug discovery [10]. Whilst current mouse models are unable to precisely recapitulate $\mathrm{AD}$-like anatomical, temporal, and spatial progression of tau pathology, they still provide powerful tools for exploring disease-modifying therapies and are widely used in $\mathrm{AD}$ drug discovery [11-13]. One of the most widely used models of tauopathy is the repressible $\mathrm{rTg}$ (tet-oTauP301L)4510 (or rTg4510) mouse [14, 15]. A significant advantage of this model is that expression of mutant tau is controllable by doxycycline administration, offering an ideal experimental positive control to determine functional effects of suppression of tau pathology. While the progression of tau pathology has been characterised thoroughly in this model [14, 15], functional decline has often been described using cross-sectional comparisons of one or two endpoints; these vary from study to study and with very little justification of why these assays were selected.

The present work aims to extend the initial phenotypic characterisation of the rTg4510 mouse model [15] and offer a detailed approach to the selection and validation of behavioural endpoints relevant for $\mathrm{AD}$ drug discovery. Male rTg4510 mice were first tested in seven different behavioural assays at an advanced pathological stage, between 12 and 15 months of age; the hypothesis was that rTg4510 mice would display robust and selective alterations in locomotor activity and spatial memory. The behavioural assays most sensitive to tau pathology were then selected and used in a separate and larger cohort of mice to assess longitudinally, from 4 to 12 months, the capacity of these endpoints to track progressive functional decline. The sensitivity of these functional endpoints to experimental modulation of tau pathology was tested in parallel via the administration of doxycycline from 4 months of age in a subgroup of $\mathrm{rTg} 4510$ mice. The hypothesis was that doxycycline would arrest the progression of tau burden and atrophy and thereby contribute to maintaining behavioural capacity at baseline levels, strengthening the hypothesis that such functional endpoints could be used to support future tau-based drug studies.

\section{Methods}

\section{Subjects and study design}

The $\mathrm{rTg}$ (tet-o-TauP301L)4510 bi-transgenic mouse model $[14,15]$ was licensed from the Mayo Clinic (Jacksonville, $\mathrm{FL}$, USA), bred on a mixed $\mathrm{FVB} / \mathrm{NCrl}+129 \mathrm{~S} 6 / \mathrm{SvEvTa}$ background by Taconic (Germantown, MD, USA), and delivered to Eli Lilly and Company (Windlesham, UK) via Envigo (Loughborough, UK). All mice were subjected to health screening, tested positive for non-MRSA Staphylococcus aureus and Proteus bacteria, and treated accordingly for 10 days with Baytril (NVSL, Stoke-on-Trent, UK) before experimental use. All subsequent animal procedures were carried out at Eli Lilly and Company Limited, in accordance with the UK Animals (Scientific Procedures) Act 1986 and with approval of the local Animal Welfare and Ethical Review Board. Mice were housed on a $12 \mathrm{~h}$ light/dark cycle with water provided ad libitum, and maintained on a foodrestricted diet at no less than $85 \%$ of their free-feeding body weight. Due to known sex differences in both the onset and severity of tau pathology in $\mathrm{rTg} 4510$ mice [16], only male mice were used for the present study. Note that all female mice were used for other studies.

Two cohorts of male rTg4510 mice were used for these studies (see Table 1). The first cohort consisted of nine bitransgenic $\mathrm{rTg} 4510$ (CC) mice and nine wild-type littermate controls (WW). These mice were tested between 12 and 15 months of age in a wide range of behavioural assays. The second cohort consisted of $93 \mathrm{CC}$ mice and $30 \mathrm{WW}$ controls. Mice were tested longitudinally, every other month from 4 to 12 months of age, in the open-field locomotor activity and T-maze rewarded alternation assays. At 4-, 8-, and 12-month time points, a subset of mice-representative of average behavioural performance of their respective treatment groups-were tested in two additional behavioural assays (Y-maze spatial novelty preference and aversive swim escape Y-maze spatial reference memory tasks) and finally perfused for pathological assessments. Ymaze assays could only be tested at these time points, and so were not tested longitudinally due to the chance that repeated testing may evoke confounding practice effects, coupled with a level of labour intensity of testing that significantly restricts throughput. Brain samples from animals at 8- and 12-month time points also underwent 
Table 1 Summary of behavioural and pathological endpoints investigated in the two cohorts of male rTg4510 mice

\begin{tabular}{|c|c|c|c|c|c|}
\hline \multicolumn{5}{|l|}{ Cohort 1} & 12-15 months \\
\hline \multicolumn{5}{|c|}{$\begin{array}{l}\text { Behaviour: spontaneous Y-maze continuous alternation, locomotor activity, Rotarod, aversive Y-maze spatial reference memory, } \\
\text { rewarded T-maze alternation, Y-maze spatial novelty preference and rewarded Y-maze visual discrimination. }\end{array}$} & $\mathrm{CC}_{(9)} \mathrm{WW}_{(9)}$ \\
\hline & $\downarrow$ \\
\hline \multicolumn{5}{|l|}{ Pathology: immunohistochemistry } & Perfused \\
\hline Cohort 2 & 4 months & 6 months & 8 months & 10 months & 12 months \\
\hline Locomotor activity & $\mathrm{CC}_{(93)} \mathrm{WW}_{(30)}$ & $C C-d_{(37)} C C_{(38)} W W_{(19)}$ & $C C-d_{(36)} C C_{(37)} W_{(19)}$ & $C C-d_{(19)} C C_{(18)} W W_{(9)}$ & $C C-d_{(19)} C C_{(18)} W W_{(9)}$ \\
\hline \multirow[t]{2}{*}{ Rewarded T-maze alternation } & $\mathrm{CC}_{(93)} \mathrm{WW}_{(30)}$ & $C C-d_{(37)} C C_{(38)} W W_{(19)}$ & $C C-d_{(36)} C C_{(37)} W W_{(19)}$ & $C C-d_{(19)} C C_{(18)} W W_{(9)}$ & $C C-d_{(19)} C C_{(18)} W W_{(9)}$ \\
\hline & $\downarrow$ & & $\downarrow$ & & $\downarrow$ \\
\hline Y-maze spatial novelty preference & $\mathrm{CC}_{(16)} \mathrm{WW}_{(11)}$ & & $C C-d_{(17)} C_{(18)} W W_{(10)}$ & & $C C-d_{(19)} C C_{(18)} W W_{(10)}$ \\
\hline \multirow[t]{2}{*}{ Aversive Y-maze spatial reference memory } & $\mathrm{CC}_{(16)} \mathrm{WW}_{(11)}$ & & $C C-d_{(17)} C C_{(18)} W W_{(10)}$ & & $C C-d_{(19)} C C_{(18)} W W_{(10)}$ \\
\hline & $\downarrow$ & & $\downarrow$ & & $\downarrow$ \\
\hline Immunohistochemistry and RT-qPCR & Perfused & & Perfused & & Perfused \\
\hline
\end{tabular}

12-month-old male rTg4510 mice from the first cohort were tested in seven behavioural assays, as ordered in the table, and then perfused for immunohistochemical assessment of tau pathology

Male mice from cohort 2 were tested from 4 to 12 months in the four pre-defined assays. Following behavioural testing in locomotor activity and T-maze alternation at 4 months, CC animals were split into two treatment groups: doxycycline (CC-d) and non-doxycycline (CC) and tested longitudinally every other month in these two assays until 12 months. At 4, 8, and 12 months, subsets of animals were tested in the Y-maze spatial reference memory and novelty preference assays after which they were all perfused for immunohistochemistry and RT-qPCR analysis

Arrows indicate animals removed from the longitudinal study and undergoing further behavioural testing and perfusion

$(n)=$ number of mice per group at time point

CC non-doxycycline treated bi-transgenic rTg4510, CC- $d$ doxycycline-treated bi-transgenic $\mathrm{rTg} 4510, R T$-qPCR reverse transcription quantitative polymerase chain reaction, $W W$ wild-type $\mathrm{rTg} 4510$

quantitative reverse transcription polymerase chain reaction (RT-qPCR). The doxycycline treatment was initiated at 4.1 months, following initial open-field locomotor activity and T-maze rewarded alternation testing. To assign animals to doxycycline treatment groups, T-maze performance of animals from the 4-month time point was ranked and then a random number generator was used to generate a sequence which allocated animals to either the doxycycline $(\mathrm{CC}+\mathrm{dox})$ or vehicle-treated (CC) control group. Reassignment of six animals was required to ensure all animals within a cage received the same treatment. $\mathrm{CC}+\mathrm{dox}$ mice received a bolus of $10 \mathrm{mg} / \mathrm{kg}$ q.d. doxycycline hyclate (Sigma Aldrich, Dorset, UK) via oral gavage for the first 2 days of treatment and were then maintained on doxycycline-mixed chow diet (Harlan Teklad Rodent Diet; $200 \mathrm{mg}$ doxycycline per $\mathrm{kg}$ of dietary chow) until the end of the study. CC and WW control groups received a bolus of a $5 \%$ glucose vehicle $(10 \mathrm{ml} / \mathrm{kg})$ via oral gavage for the first 2 days of treatment and were then maintained on standard chow. The dietary composition of doxycyclinemixed and standard chow was similar for each group.

\section{Behaviour}

\section{Motor activity and motor co-ordination tasks}

Open-field locomotor activity Spontaneous locomotor activity was assessed in clear Perspex open-field arenas $(40 \times 40 \times 30 \mathrm{~cm})$ under complete darkness. Arenas were based on infrared fields $(100 \times 100 \mathrm{~cm})$ with four boxes placed on each field and monitored using overhead infrared cameras (Sanyo VCV-3412P, Tracksys Ltd., UK). Cameras fed into a Quad compressor unit (Sanyo VDM-801P, Tracksys Ltd., UK) which relayed data to a computer running the image analysis software Ethovision XT v8.5 (Noldus, Netherlands). Mice were allowed to freely explore the arena for $60 \mathrm{~min}$ during which time the distance moved was measured as a total over $60 \mathrm{~min}$ (primary outcome measure) and in 5-min time bins to discern progressive changes in activity/habituation (secondary outcome measure).

Rotarod Motor co-ordination was assessed in a fivestation mouse Rotarod (ENV 575MA, Med Associates Inc., USA). Mice were placed on a slowly rotating drum and their ability to remain on the drum was assessed for up to $5 \mathrm{~min}$. An accelerating speed protocol was utilised whereby rotation speed gradually increased from $4 \mathrm{rpm}$ to a maximum of $40 \mathrm{rpm}$ over the 5 -min test period. Fall latency (primary outcome measure) was recorded over three separate trials and is reported here as an average of the three trials. There was an inter-trial interval (ITI) of approximately 5-10 min to allow mice to recover. In some cases, mice were able to cling to the rotating beam for one full revolution; this was registered as a fall by the experimenter and the trial was ended.

\section{Short-term habituation and spatial working memory tasks}

Y-maze spontaneous, continuous alternation Y-maze arenas were based on infrared fields $(100 \times 100 \mathrm{~cm})$ and movement data captured as described in the open-field 
locomotor activity test. Each Y-maze consisted of three equal arms $(30 \times 8 \times 20 \mathrm{~cm})$, spaced $120^{\circ}$ apart. Arms were assigned arbitrary labels of $\mathrm{A}, \mathrm{B}$, and $\mathrm{C}$. Test sessions were $15 \mathrm{~min}$, and began as soon as the mouse was placed in the arena. Mice were placed in the distal end of one arm, facing away from the centre of the maze and allowed to freely explore all arms of the Y-maze. Initial data acquisition was conducted by Ethovision XT v8.5 (Noldus, Netherlands) and then subject to further processing by in-house software (Ethovision Collection, Handling and Ordering v2.2.0.0, Lilly Research Laboratories, UK). The number of arm entries and nonrepeating arm entry triads (e.g. $\mathrm{ABC}, \mathrm{CAB}$ ) were recorded, from which percentage alternation (primary outcome measure), as well as total distance moved (secondary outcome measure) were derived.

Y-maze spontaneous, spatial novelty preference Ymaze arenas, infrared fields, and the video tracking system used were identical to those described for the continuous alternation task. Spatial novelty preference was assessed in two separate phases, an exposure phase and a test phase. Mice were pseudo-randomly assigned two arms (the "start arm" and the "other/familiar arm") to which they were exposed and allowed to freely explore for $5 \mathrm{~min}$ ("exposure" phase). The entrance to the third "novel" arm was blocked by the presence of a large opaque insert. At the end of the 5-min period, the mouse was removed from the maze and placed back in its home cage for $1 \mathrm{~min}$, following which the "test" phase started. The insert was removed; mice were placed back into the start arm and allowed to explore the entire maze (i.e. all three arms) for $2 \mathrm{~min}$. Videos were recorded and manually scored offline using Abacus v2.0 (Lilly Research Laboratories, UK). A discrimination ratio of the time spent in the novel arm (primary outcome measure: time in novel arm/(time in novel + familiar arms)) was calculated for the test phase. The amount of time spent in each arm as well as the number of arm entries (co-secondary outcome measures) were recorded during both exposure and test phases.

T-maze rewarded alternation Discrete-trial rewarded alternation was tested using a semi-automated T-maze (Apogee Engineering Analysis Solutions, Norwich, UK). This apparatus was constructed of matte black, $8-\mathrm{cm}$ wide Perspex with $20-\mathrm{cm}$ high transparent Perspex walls. The external lengths of maze edges were $86 \mathrm{~cm}$ (choice end), $105 \mathrm{~cm}$ (return arm) and $22.5 \mathrm{~cm}$ (delay end). The centre arm was $83 \mathrm{~cm}$ in length and a door was located $63 \mathrm{~cm}$ from the choice point forming a holding area at the base of the start arm. The entry of an animal into specific areas of the maze was detected using infrared beam breaks and passed to a microcontroller (Arduino
Mega 2560). Custom-made Matlab programs automatically controlled the maze doors and test procedure, allowing it to run without human intervention. Rewards were delivered by three pellet dispensers, one located at the end of each reward arm and a third in the delay/holding area of the maze to encourage return of the animal to the starting point for the subsequent test phase or trial. Mice were trained following a two-stage protocol: forced alternation training and discrete-trial rewarded alternation testing. During the forced alternation training stage, mice were released from the holding area at the base of the T-maze, allowed to run along the centre-arm and forced to turn toward one of the reward areas to receive a sucrose pellet reward. Mice then returned to the holding area to collect a second reward pellet, after which another forced trial was initiated. At this stage, a 0-s ITI was used, and mice were trained for a maximum of 60 trials or 30 min daily. Once most mice were performing 40 training trials or more in a session, mice were moved on to the discrete-trial rewarded alternation protocol. Each trial consisted of two phases-a sample phase and a test phase. During the sample phase, mice were forced to turn toward the left or right arm and return to the starting/holding area. Two reward pellets were collected along the way, one at the end of the choice arm and one in the holding area. During the test phase of each trial, mice were allowed to choose between the two arms of the T-maze but were rewarded when visiting the novel arm only (i.e. arm not explored during the sample phase). Forced left or right allocations during the sample phase were pseudo-randomized with no more than three consecutive sample runs to the same side. Mice were allowed to run for a maximum of 20 trials or $30 \mathrm{~min}$ daily over 3 days of testing. A 2-s ITI and a 5-s sampleto-test delay were used. The percentage of correct choices (primary outcome measure: number of correct choice/number of trials) as well as the choice latency (secondary outcome measure) were recorded and calculated for each animal.

\section{Spatial reference memory task and visual discrimination control task}

Aversive (swim escape) Y-maze spatial reference memory Y-maze arenas were identical to those used for continuous alternation and spatial novelty preference tasks. Extra-maze cues were placed around the room. An escape platform $(8 \times 8 \times 11 \mathrm{~cm})$ was placed at the end of one arm (i.e. the goal arm) and the maze was flooded to a depth of approximately $12 \mathrm{~cm}$ so that the platform was submerged. White tempera powder paint (Educational Colours Pty Ltd., Australia) was added as an opacifier to ensure the platform was not visible. Spatial reference learning was assessed over 8 days of 
testing, with five trials per day and an ITI of approximately 5-10 min. Mice were pseudo-randomly assigned a goal arm, defined according to its allocentric position relative to the room cues and therefore constant during the entire testing phase. Goal arms were counterbalanced with respect to treatment group such that approximately equal numbers of mice were trained to each of the three arms. The other two arms or start arms were used in a pseudo-randomised order; these were counterbalanced to ensure that, over a 10-trial block, each start arm was used five times and no more than three times consecutively. For each trial, mice were gently placed at the distal end of either start arm, facing away from the centre of the maze and allowed to swim freely to either of the other two arms. A correct choice was scored when mice entered the goal arm, after which they were allowed to spend $30 \mathrm{~s}$ on the escape platform before being removed from the maze. If mice chose incorrectly and entered the other arm, they were punished with $30 \mathrm{~s}$ of forced swim, during which entry to the goal arm was blocked. Arm entry was classed as all four paws crossing into an arm. Mice who failed to make a choice after $60 \mathrm{~s}$ had elapsed were removed from the maze and the trial was skipped. Twenty-four hours after the final day of spatial learning, a probe test was conducted to test for spatial reference memory. The escape platform was removed from the arena. Mice were placed in one of the two start arms and allowed $30 \mathrm{~s}$ of free exploration of the maze, starting when mice exited the start arm. The percentage of correct choices and percentage time spent in the goal arm (co-primary outcome measures) were calculated for the spatial learning and memory probe test, respectively.

Y-maze rewarded visual discrimination Y-maze arenas used were identical to that used for the previous Y-maze tasks. For each trial, the Y-maze had a start arm and two goal arms made visually distinct by adding floor inserts; one painted grey and one black and white striped. An opaque white food well was placed at the end of each of the three arms. Mice were first familiarized to the maze without floor inserts and in a separate room to the testing room. Multiple sucrose pellets were placed in each of the food wells. Mice were left to explore the maze until they readily ate all pellets. Following this, mice were trained to discriminate between the two floor inserts to locate the goal/rewarded arm over 10 days of testing and 10 trials per day, with an ITI of approximately $10 \mathrm{~min}$. Mice were pseudo-randomly assigned either one of the two patterns as a goal arm in a counterbalanced manner between groups. The start arm as well as the left/right location of the floor inserts and thus the goal arm location varied from trial to trial (i.e. there was no spatial solution), and was determined according to a pseudo-random sequence (with equal numbers of right/left presentation and no more than three consecutive trials with the same correct location). The maze was rotated $120^{\circ}$ clockwise at random intervals, making sure spatial and olfactory cues could not reliably be used to solve the task. For each trial, the food well in the goal arm was baited with a sucrose pellet reward, and mice were placed at the distal end of the start arm, facing outwards. A correct choice was scored when mice entered the assigned/correct goal arm directly. If mice chose incorrectly they were immediately removed from the maze and then returned to their home cage. A choice was classed as all four paws being placed in either of the non-start arms. The percentage of correct choices (primary outcome measure) was calculated and used as an index of discrimination learning.

\section{Pathology}

\section{Perfusion and brain harvesting}

Animals were terminally anaesthetised with pentobarbital administered via intraperitoneal injection and transcardially perfused with saline. Brains were then removed and weighed before 10-20 mg of rostral cortex tissue was dissected and snap-frozen in RNase-free tubes for RT-qPCR analysis. The remaining brain tissue was immersion fixed in $10 \%$ buffered formalin for immunohistochemical analyses

\section{Quantitative reverse transcription $P C R$}

Expression of the transgenic tau was analysed in samples taken at 8 and 12 months by RT-qPCR following standard techniques. Total RNA was isolated from snap-frozen samples of cortex using the Ambion RNAqueous 4 PCR kit (Thermofisher Scientific, UK) and following the manufacturer's protocol; DNAse digestion was performed to remove any contaminating DNA. RNA was quantified using a Nanodrop spectrophotometer (Thermofisher Scientific, UK) and its quality assessed by running an aliquot on an Agilent Nano 6000 Chip on an Agilent 2100 Bioanalyzer (Agilent Technologies, Stockport, UK). cDNA was synthesised from 36 ng of RNA using random hexamer primers $(2.5 \mu \mathrm{M})$ and Moloney Murine Leukemia Virus Reverse Transcriptase $(1.25 \mathrm{U} / \mu \mathrm{l})$ in reaction buffer containing dATP, dCTP, dTTP, dGTP (0.5 $\mu \mathrm{M}$ of each), $\mathrm{MgCl}_{2}$ $(5.5 \mathrm{mM})$, and RNase inhibitor $(0.4 \mathrm{U} / \mu \mathrm{l})$ (all reagents from Thermofisher Scientific, UK). The reverse transcription was incubated at $25{ }^{\circ} \mathrm{C}(10 \mathrm{~min}), 48{ }^{\circ} \mathrm{C}(30 \mathrm{~min})$, and $95{ }^{\circ} \mathrm{C}$ (5 min). Primers for qPCR were synthesised by Eurofins Genomics (Ebersberg, Germany) according to the sequences described by Santacruz et al. [14]: human tau transgene: forward 5'-CCC AAT CAC TGC CTA TAC CC3'; reverse 5'-CCA CGA GAA TGC GAA GGA-3'; GAPDH: forward 5'-TGG TGA AGC AGG CAT CTG AG-3'; reverse 5'-TGC TGT TGA AGT CGC AGG AG-3'; 
mouse tau: forward 5'-AGC CCT AAG ACT CCT CCA-3'; reverse 5'-TGC TGT AGC CGC TTC GTT CT-3'. Quadruplicate qPCR reactions were assembled and aliquoted robotically into a 96-well reaction plate such that each $5-\mu \mathrm{l}$ reaction contained $2.5 \mu \mathrm{l} \mathrm{SYBR}{ }^{\circ}$ Green mastermix (QuantiTect SYBR Green PCR Kit, Qiagen, Manchester, UK), 225 pg cDNA, and appropriate primer as follows: $400 \mathrm{nM}$ transgenic tau; $200 \mathrm{nM}$ mouse tau; $200 \mathrm{nM}$ GAPDH. The qPCR reaction was run on an ABI 7900HT Fast Real-Time PCR System using a two-stage anneal and elongation protocol with the following parameters: $50{ }^{\circ} \mathrm{C} 2 \mathrm{~min}, 95^{\circ} \mathrm{C}$ $10 \mathrm{~min} ; 95^{\circ} \mathrm{C} 15 \mathrm{~s}, 58{ }^{\circ} \mathrm{C} 1 \mathrm{~min}\left(40\right.$ cycles); $95^{\circ} \mathrm{C} 15 \mathrm{~s}, 58^{\circ}$ $\mathrm{C} 15 \mathrm{~s}, 95{ }^{\circ} \mathrm{C} 15 \mathrm{~s}$. Data were analysed using the $\Delta \Delta \mathrm{Ct}$ method using GAPDH as the reference gene and the nondoxycycline treated group as the calibrator. These analyses were performed in a blinded fashion.

\section{Immunohistochemistry}

Brain samples were processed using the Tissue TEK VIP processor (GMI Inc., Ramsey, MN, USA) before being embedded in paraffin wax for coronal brain sectioning. Serial sections $(6 \mu \mathrm{m})$ were taken using HM 200 and HM 355 rotary microtomes (Thermo Scientific Microm, Germany). Immunohistochemistry (IHC) was performed using a primary antibody for tau phosphorylated at serine 409 (PG-5, $0.11 \mu \mathrm{g} / \mathrm{ml}$ from Peter Davies; Albert Einstein College of Medicine, Bronx, NY, USA) as previously described [17]. Stained sections were digitised using the Scanscope XT slide scanner (Aperio, CA, USA) at $20 \times$ magnification. Imagescope software (version 11.1.2.760; Aperio) was used to view the digitized tissue sections and delineate the regions of interest (ROIs) which included the hippocampus and cortex. PG-5 positive tau pathology was quantified in these ROIs using a positive pixel algorithm that was calibrated to detect only intensely labelled tau pathology, to avoid any non-specific staining. The burden of tau pathology was expressed as percentage area, and the ROI area $\left(\mathrm{mm}^{2}\right)$ was used as a marker of atrophy. These analyses were performed in a blinded fashion.

\section{Statistics}

All datasets were checked for normality and homogeneity of variance and transformed as appropriate, before subsequent statistical analyses were conducted using Statistica v13.0 (Statsoft Ltd., Bedford, UK). Analysis of variance (ANOVA) was used to analyse behavioural and pathological data. One-way ANOVA was used for all the crosssectional assessments. Repeated measures ANOVA was used for analysis of the longitudinal assessments, using Proc Mixed of the SAS system (SAS/STAT v14.1, SAS Institute Inc., USA). Genotype/treatment group (WW, CC, and $\mathrm{CC}+\mathrm{dox}$ ) was used as the main between-subject factor, and age, time and trial block were used as within- subject factors as appropriate. Significant main effects or interactions were subsequently explored with appropriate post hoc analyses and $p$ values corrected for multiple comparisons. Spearman's rank correlation coefficient analyses were used to assess the relationship between effects on pathology vs. behaviour, as well as pathology vs. pathology and behaviour vs. behaviour. Correlation coefficients and unadjusted $p$ values are reported.

\section{Results}

Study 1: Selection of cognitive and behavioural endpoints in 12- to 15 -month-old male $\mathrm{rTg} 4510$ mice

\section{Missing and excluded data}

Four mice (three CC and one WW) were excluded from the analysis of T-maze rewarded alternation data as these animals failed to complete a minimum of five trials. One mouse $(\mathrm{CC})$ was removed from spatial novelty preference analysis as it failed to leave the start arm and explore the rest of the maze during the 5-min exposure phase.

\section{Severe tau pathology and brain atrophy in old male rTg4510 CC mice}

Widespread tau pathology was observed in coronal brain sections taken from 15-month-old male rTg4510 CC mice (Fig. 1a). PG-5-positive tau pathology was severe in the hippocampus $(10.7 \pm 0.3 \%$ burden) and cortex (16.6 $\pm 0.7 \%$ ) of CC mice (Fig. 1b). Extensive atrophy was also observed, as indicated by significant reductions in the hippocampal and cortical area compared to age-matched WW littermate controls (hippocampus: $\mathrm{F}_{1,16}=55.26, p<$ 0.001; cortex: $\left.\mathrm{F}_{1,16}=44.57, p<0.001\right)$.

\section{Altered motor activity but intact motor co-ordination in old male rTg 4510 CC mice}

Old CC mice were consistently more active than agematched WW controls over $60 \mathrm{~min}$ of open-field spontaneous exploration, exhibiting no habituation over this time period (Fig. 1c; genotype: $F_{1,16}=66.71, p<0.001$; time: $\mathrm{F}_{23,368}=11.15, p<0.001$; genotype $\times$ time: $\mathrm{F}_{23,368}=$ $7.45, p<0.001)$. Despite this profound hyperactive behaviour, motor co-ordination of CC animals remained intact as demonstrated by the comparable performance between genotypes on the Rotarod task (Fig. 1d; genotype: $\mathrm{F}_{1,15}=1.25, p=0.28$ ).

\section{Impaired spatial reference memory but not visual cue discrimination in old male rTg4510 CC mice}

Spatial reference learning was severely impaired in old CC mice compared to WW controls, as illustrated by lower percent correct choices in three out of the four training blocks (Fig. 1e; genotype: $\mathrm{F}_{1,16}=19.19, p$ $<0.001$; block: $\mathrm{F}_{3,48}=10.98, \quad p<0.001$; genotype $\times$ block: $\left.F_{1,16}=3.02, \quad p=0.04\right)$. The spatial reference memory of CC animals was also impaired, as 

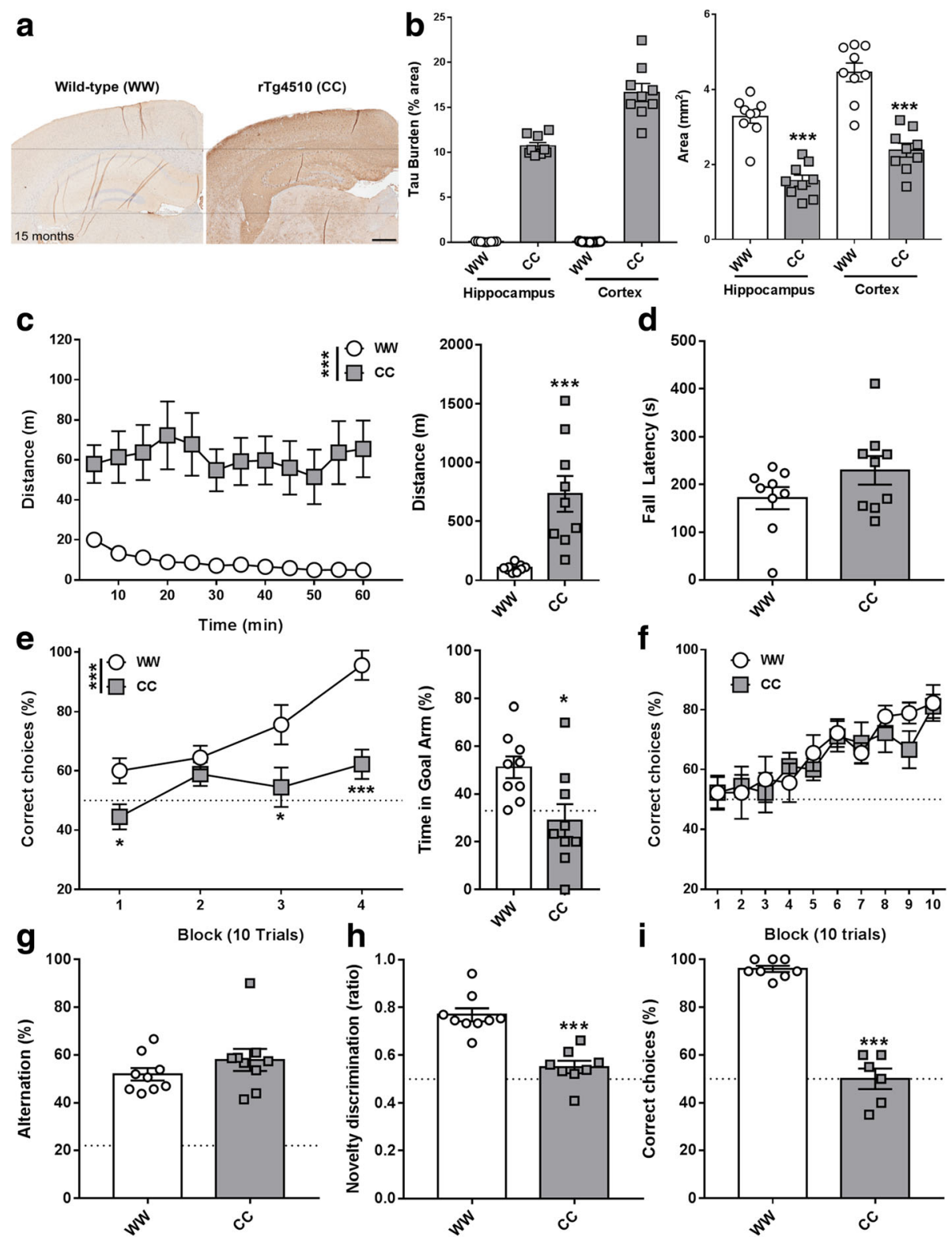

Fig. 1 Immunohistochemical and behavioural profiling of 12- to 15-month-old rTg4510 mice. Pathology: Bi-transgenic rTg4510 (CC) mice displayed severe hippocampal and cortical tau burden and atrophy (a), as measured by PG-5-positive staining and area, respectively (b). Behaviour: CC mice displayed profound hyperactive behaviour in the open-field locomotor activity task (c), whereas motor co-ordination remains intact as measured in the Rotarod task (d). CC mice were impaired in the acquisition and 24-h probe testing in the swim escape Y-maze spatial reference memory task (e). Acquisition of a Y-maze non-spatial, visual cue discrimination learning task was no different than in wild-type/non-transgenic rTg4510 (WW) controls (f). No deficit was observed in the Y-maze continuous alternation task (g), while CC mice were impaired in both the spatial novelty preference (h) and discrete-trial rewarded alternation (i). Scale bar $=500 \mu \mathrm{m}$. All data are presented as mean $\pm \mathrm{SEM}$, dotted lines denote chance level. ${ }^{*} p<0.05,{ }^{* *} p<0.01$, ${ }^{* * *} p<0.001$, versus WW controls

suggested by the decrease in the time spent exploring the goal arm during the 30 -s probe test conducted $24 \mathrm{~h}$ after the last training session (Fig. 1e; genotype: $\mathrm{F}_{1,16}=7.33, p=0.02$ ). Whilst age-matched WW controls performed significantly above chance level, CC mice did not (33\% chance level; WW vs. chance: $\mathrm{t}_{8}=$ 3.92, $p=0.004 ; C C$ vs chance: $\left.\mathrm{t}_{8}=-0.64, p=0.54\right)$.
Despite profound deficits in spatial reference learning and memory, basic visual cue discrimination learning was intact in $\mathrm{CC}$ mice as shown by the steady and comparable increase in percent correct choices across training blocks in both $\mathrm{CC}$ and WW groups (Fig. 1f; genotype: $\mathrm{F}_{1,16}=0.22, p=0.64$; block: $\mathrm{F}_{9,144}=8.06, p<$ 0.001 ; genotype versus block: $\mathrm{F}_{9,144}=0.52, p=0.86$ ). 
Impaired short-term habituation and spatial working memory in old male rTg4510 CC mice

Short-term habituation and spatial working memory were assessed using three different but complementary maze tasks. rTg4510 mice were first tested in a Y-maze spontaneous continuous alternation task. CC performance was similar to that of WW controls and significantly above alternation chance level (Fig. 1g; genotype: $\mathrm{F}_{1,16}=1.31, p=0.27 ; \mathrm{WW}$ vs. chance: $\mathrm{t}_{8}=11.46, p<$ 0.001 ; $C$ vs. chance: $\left.t_{8}=7.81, p<0.001\right)$. Mice were then tested in a Y-maze spontaneous spatial novelty preference task. CC mice performed poorly compared to WW controls, with a lower and close-to-chance novelty discrimination ratio (Fig. 1h; genotype: $\mathrm{F}_{1,15}=33.27, p<$ 0.001; WW vs. chance: $t_{8}=9.93, p<0.001 ; C C$ vs. chance: $\left.t_{7}=1.96, p=0.09\right)$. Mice were finally tested in a T-maze discrete-trial rewarded alternation task. CC mice were again impaired relative to WW controls, with alternation performance no different than chance level (Fig. 1i; genotype: $\mathrm{F}_{1,12}=135.41, p<0.001$; WW vs. chance: $t_{7}=35.52, p<0.001$; CC vs. chance: $t_{5}=0.00, p$ $=1.00)$. Pathology at this time point was so extensive that no $\mathrm{CC}$ animal was able to perform above the chance level in T-maze performance, and all but one CC mice displayed a robust hyperactive phenotype in open-field locomotor activity.

\section{Study 2: Validation of selected endpoints in 4- to 12- month-old, doxycycline-treated male rTg4510 mice Missing and excluded data}

Five mice (two CC and three CC + dox) were culled due to poor health during the 8-month study; these animals were removed from all behavioural and pathological analyses. One mouse (CC) was excluded from spatial novelty preference analysis as it was able to escape the arena during testing. Five animals (one WW, three CC, and one $C C+$ dox $)$ repeatedly failed to complete more than five trials during T-maze testing and were therefore excluded from T-maze longitudinal analysis. A further two mice (both WW) were removed from T-maze analysis at specific time points where they completed fewer than five trials, but not from the entire analysis.

\section{Doxycycline arrested the progression of both tau burden and brain atrophy}

Progressive pathological changes were seen in the brains of 4-, 8-, and 12-month-old male rTg4510 mice that appeared to be preserved by doxycycline treatment initiated at 4.1 months (Fig. 2a). This occurred despite only a $40-50 \%$ reduction in tau expression compared to CC animals as measured by RT-qPCR analysis (Fig. 2b; 8 months: $\mathrm{F}_{1,40}=184.51, p<0.001 ; 12$ months: $\mathrm{F}_{1,42}=$ 93.73, $p<0.001)$.
At 4 months of age, tau burden in the hippocampus $(2.1 \pm 0.2 \%)$ and cortex $(3.6 \pm 0.4 \%)$ of CC mice was relatively low (Fig. 2d and e). Signs of brain atrophy were apparent at this stage; whole brain weight was significantly reduced, which coincided with reductions in hippocampal and cortical area (Fig. 2c, f, and g; brain weight: $\mathrm{F}_{1,25}=8.92, p=0.006$; hippocampus size: $\mathrm{F}_{1,25}=$ $42.80, p<0.001$; cortex size: $\mathrm{F}_{1,25}=0.57, p=0.46$ ). By 8 and 12 months of age, significant increases in tau burden were observed in both the hippocampus $(7.3 \pm 0.6 \%$ and $10.6 \pm 0.7 \%$, respectively) and cortex $(10.0 \pm 0.7 \%$ and $16.0 \pm 1.1 \%$, respectively). Widespread atrophy was also observed; brain weight was again smaller in 8-month CC mice, and appeared to decrease further at the later time point of 12 months (Fig. 2c; 8 months: $\mathrm{F}_{2,42}=7.93, p<$ $0.001 ; 12$ months: $\left.\mathrm{F}_{2,42}=101.85, p<0.001\right)$. Progressive decreases in size were observed in both hippocampal (Fig. 2f; 8 months: $\mathrm{F}_{2,40}=29.33, p<0.001 ; 12$ months: $\mathrm{F}_{2,42}=74.44, p<0.001$ ) and cortical (Fig. 2g; 8 months: $\mathrm{F}_{2,42}=22.92, \quad p<0.001 ; 12$ months: $\mathrm{F}_{2,42}=61.36, p<$ $0.001)$ areas compared to WW controls.

Despite only moderate levels of transgene suppression at 8 and 12 months, hippocampal $(1.9 \pm 0.6 \%$ and $2.6 \pm$ $0.7 \%$, respectively) and cortical $(3.2 \pm 0.7 \%$ and $5.9 \pm$ $1.1 \%$, respectively) tau burden were significantly decreased in $\mathrm{CC}+$ dox mice compared to $\mathrm{CC}$ controls (Fig. 2d and e; hippocampus, 8 months: $\mathrm{F}_{2,40}=31.96, p<$ 0.001, 12 months: $\mathrm{F}_{2,42}=53.16, p<0.001$; cortex, 8 months: $\mathrm{F}_{2,42}=43.43, p<0.001,12$ months: $\mathrm{F}_{2,42}=$ $42.20, p<0.001)$, levels that were largely comparable to those observed at 4 months. Doxycycline also prevented further brain atrophy, as illustrated by the significant differences in $\mathrm{CC}+$ dox vs. $\mathrm{CC}$ brain weight (Fig. 2c; 8 months: $\mathrm{F}_{2,42}=7.93, p=0.72 ; 12$ months: $\mathrm{F}_{2,42}=$ 101.85, $p<0.001$ ), hippocampus area (Fig. 2f; 8 months: $\mathrm{F}_{2,40}=29.34, \quad p=0.02 ; 12$ months: $\mathrm{F}_{2,42}=74.44, p<$ 0.001 ), and cortex area (Fig. $2 \mathrm{~g} ; 8$ months: $F_{2,42}=22.92$, $p<0.001 ; 12$ months: $\left.\mathrm{F}_{2,42}=61.36, p<0.001\right)$.

\section{Doxycycline prevented further decline in behavioural and cognitive function}

The pattern of changes observed from 4 to 8 and 12 months was similar across all four behavioural assays. The performance of male $\mathrm{rTg} 4510 \mathrm{CC}$ mice progressively worsened over time, reaching levels similar to those observed in 12- to 15-month-old CC mice during study 1 . Doxycycline treatment from 4.1 months prevented further functional decline and showed trends towards reversing initial behavioural deficits.

A progressive increase in open-field locomotor activity was observed over time in CC mice, whilst activity in WW littermates remained stable (Fig. 3a; group: $\mathrm{F}_{2,108}=$ $31.09, p<0.001$; age: $\mathrm{F}_{4,73}=19.80, p<0.001$; group $\times$ age: $\left.\mathrm{F}_{8,99}=6.16, p<0.001\right)$. CC mice were hyperactive from 
a

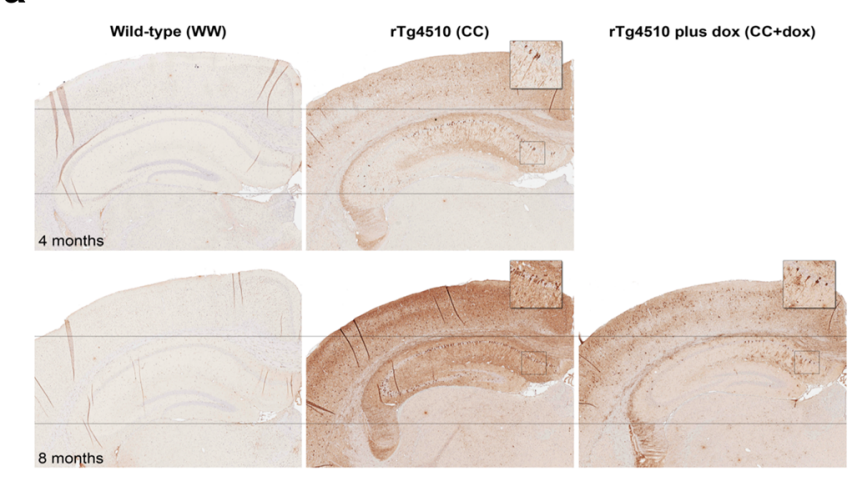

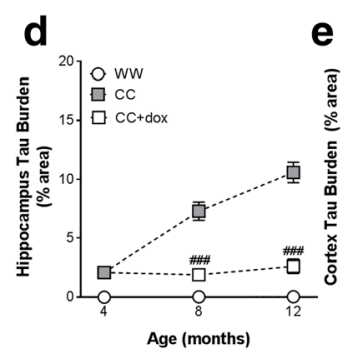

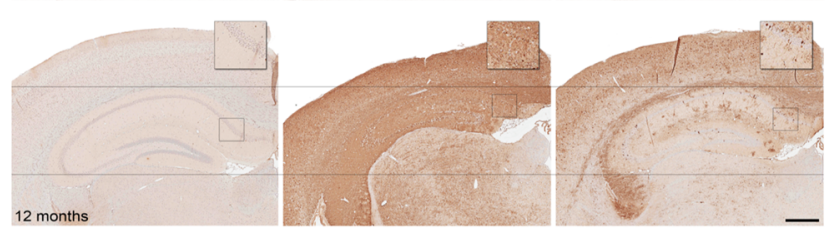

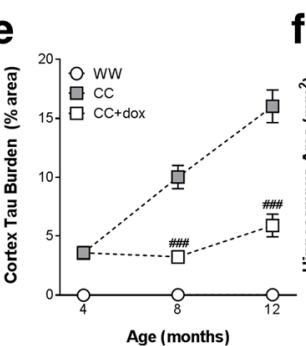

f b

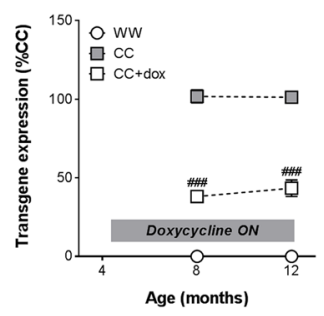

C

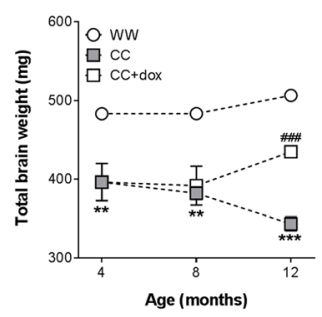

g

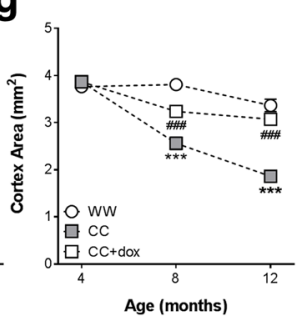

Fig. 2 Tau pathology in male rTg4510 mice following doxycycline treatment. Representative immunohistochemical images of 4-, 8-, and 12-month-old male rTg4510 brains (a). RT-qPCR revealed a 40 to 50\% reduction of tau expression in bi-transgenic $r \operatorname{Tg} 4510$ (CC) mice receiving doxycycline (dox) treatment (b). Brain weight was decreased in CC mice at all time points, and was attenuated in 12-month-old CC + dox mice (c). Increasing levels of tau pathology were observed in both the hippocampus (d) and cortex (e) of CC mice from 8 months of age. These were normalised following doxycycline treatment. CC mice displayed high levels of atrophy in the hippocampus at all time points ( $\mathbf{f}$ ) and in the cortex at 8 and 12 months (g); atrophy was prevented by doxycycline treatment. Note that hashed lines do not represent longitudinal, repeated assessment of these animals. Scale bar $=500 \mu \mathrm{m}$. All data are presented as mean \pm SEM. ${ }^{*} p<0.05,{ }^{* *} p<0.01,{ }^{* *} p<0.001$, versus wild-type/non-transgenic rTg4510 (WW) controls; ${ }^{\#} p<0.05,{ }^{\# \#} p<0.01,{ }^{\# \#} \# p<0.001$, versus rTg4510 CC mice

4 months of age ( $p=0.003$ versus WW) and became progressively more active at later time points (6-12 months: all $p<0.001$, versus WW). Doxycycline treatment prevented further increases in locomotor activity in CC mice, with CC + dox mice significantly less active than non-doxtreated counterparts across the 8- to 12-month age period (8-12 months: all $p<0.001$, versus CC).

$\mathrm{T}$-maze rewarded alternation performance was also progressively impaired in $\mathrm{CC}$ mice (Fig. 3b; group: $\mathrm{F}_{2,85}$ $=41.47, p<0.001 ;$ age: $\mathrm{F}_{4,65}=3.99, p=0.006$; group $\times$ age: $\left.\mathrm{F}_{8,88}=6.60, p<0.001\right)$. Significant deficits were present at 4 months in $\mathrm{CC}$ mice which declined to chance level by 12 months (4-12 months: all $p<0.001$, versus WW). In contrast, WW mice exhibited stable performance over this time period, while $\mathrm{CC}+$ dox mice displayed significantly elevated performance from 6 months of age (6 months: $p=0.03$; $8-12$ months: $p<$ $0.001, \mathrm{CC}+$ dox versus $\mathrm{CC})$.
Spatial novelty discrimination in 4-month-old CC mice was impaired compared to WW controls (Fig. 3c; group: $\mathrm{F}_{1,25}=14.16, p<0.001$ ); $\mathrm{CC}$ performance was no different than chance level. At 8 months, however, no significant difference was observed between all three groups (WW, CC, and CC + dox group: $\mathrm{F}_{2,42}=2.17, p=$ 0.13 ). Finally, at 12 months, novelty discrimination performance of $\mathrm{CC}$ mice was again impaired compared to the other two groups of mice (group: $\mathrm{F}_{2,42}=11.86, p<$ 0.001). Planned comparisons revealed a significant decrease in novelty discrimination in CC animals $(p<$ 0.001 , versus $\mathrm{W} W$ ), which was attenuated by doxycycline treatment $(p=0.002, \mathrm{CC}+$ dox versus $\mathrm{CC})$.

Spatial reference learning and memory declined over time in CC mice (Fig. 3d; 4 months: $\mathrm{F}_{1,25}=5.05, p=0.03$; 8 months: $\mathrm{F}_{2,42}=6.21, p=0.004 ; 12$ months: $\mathrm{F}_{2,41}=$ $19.32, p<0.001$, versus WW), with performance at levels no different than chance by 8 months of age. Treatment 

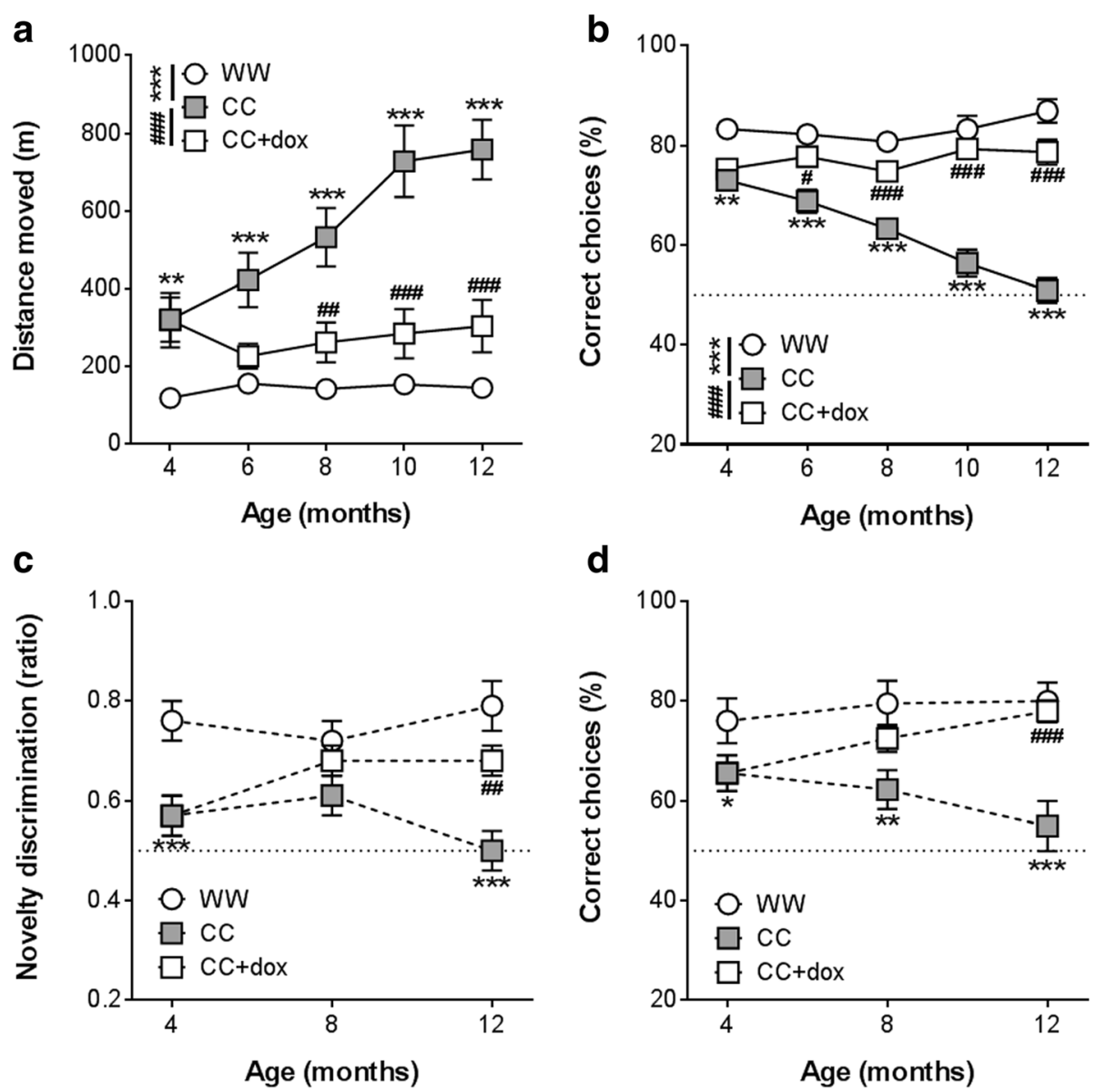

Fig. 3 Behavioural alterations in male rTg4510 mice following doxycycline treatment. Locomotor activity revealed bi-transgenic rTg4510 (CC) mice to be hyperactive at 4 months and become increasingly more active as they age, whilst CC + doxycycline (dox) mice remained stable at levels similar to those observed prior to starting doxycycline treatment (a). T-maze rewarded alternation task accuracy was significantly poorer in CC mice at 4 months of age, and declined progressively until reaching chance levels at 12 months. Doxycycline treatment significantly improved performance; CC + dox mice made significantly more correct choices than CC mice from 6 months onwards (b). CC mice were impaired in novelty discrimination, as assessed in a spatial novelty preference Y-maze task. CC + dox mice showed improved novelty discrimination compared to CC at 12 months (c). Spatial learning was assessed using acquisition of an aversively-motivated Y-maze; CC mice made significantly fewer correct choices over 40 trials than WW mice, and further decreases were seen with age. Doxycycline treatment improved performance, with CC + dox mice making more correct choices than CC at 8 and 12 months (d). Note that hashed lines do not represent longitudinal, repeated assessment of these animals. All data presented as mean \pm SEM, dotted horizontal lines denote chance level. ${ }^{*} p<0.05,{ }^{* *} p<0.01,{ }^{* *} p<0.001$, versus wild-type/ non-transgenic rTg4510 (WW) controls; ${ }^{*} p<0.05,{ }^{\# \#} p<0.01,{ }^{\# \# \#} p<0.001$, versus rTg4510 CC mice

with doxycycline from 4.1 months was able to prevent this decline at both 8 and 12 months $\left(8\right.$ months: $\mathrm{F}_{2,42}=$ 6.21, $p=0.017 ; 12$ months: $\mathrm{F}_{2,41}=19.32, p<0.001$, versus $\mathrm{CC})$, effectively restoring performance to levels that were not significantly different to those of WW animals.

\section{Relationships between measures of pathology and/or behaviour}

Spearman's rank correlation analyses of the multiple study endpoints revealed strong relationships between measures of tau pathology and brain atrophy in CC mice, but not $\mathrm{CC}+$ dox mice (data not shown). In CC mice, hippocampal and cortical tau burden were negatively correlated with hippocampal and cortical area, respectively (Fig. 4a; hippocampus: $r=-0.70, p<0.001$; cortex: $r=-0.69, p<0.001)$. Moderate relationships were also observed between various behavioural endpoints. Open-field locomotor activity correlated with all three other behavioural endpoints: T-maze rewarded alternation (Fig. 4b; $r=-0.52, p<0.001$ ), Y-maze novelty preference $(\mathrm{r}=-0.41, \quad p=0.01)$, and aversive Y-maze acquisition $(r=-0.41, p<0.01)$. CC mice also showed strong correlations between behavioural and pathological outcomes (Fig. 5). Locomotor activity and Tmaze alternation both correlated strongly with all measures of pathology investigated. Aversive Y-maze acquisition correlated with hippocampal and cortical area, but Y-maze novelty preference did not. 

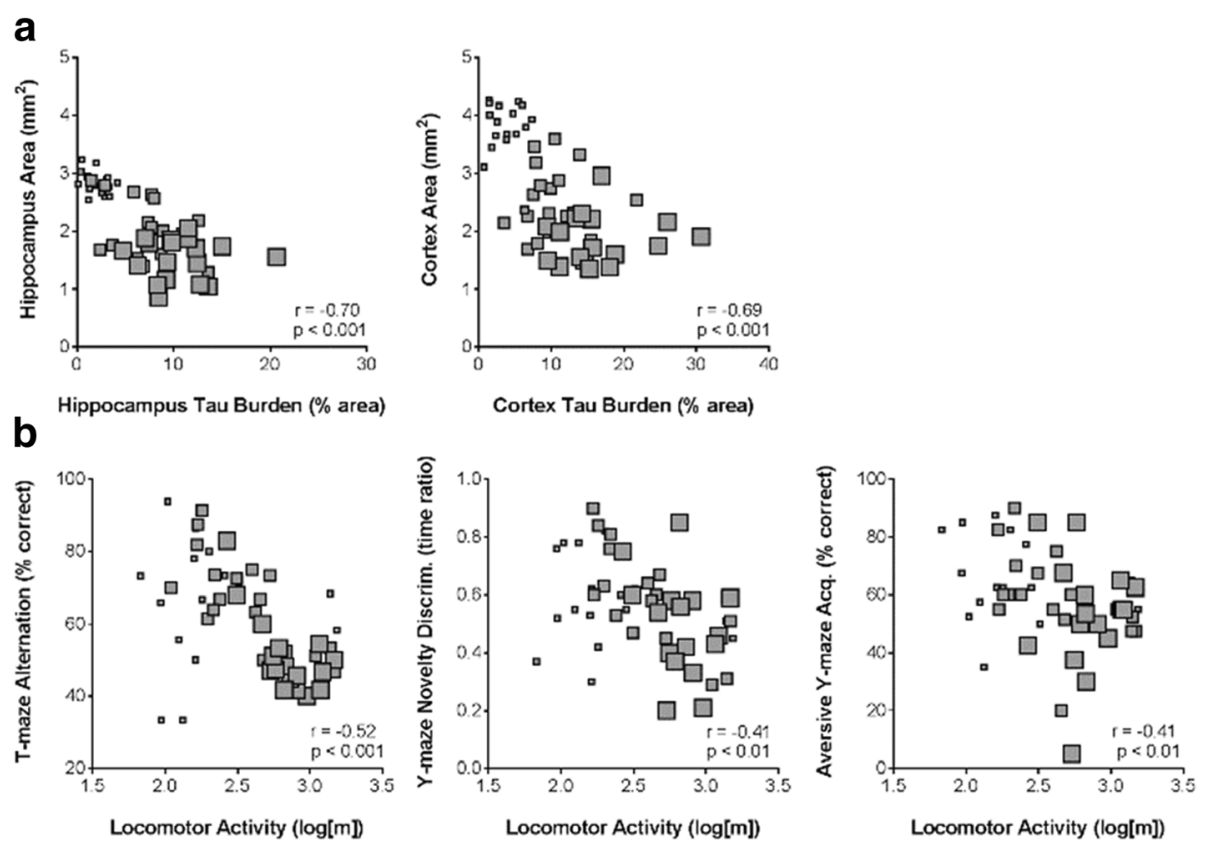

Fig. 4 Relationships between measures of pathology or behaviour in male rTg4510 CC mice. Strong negative correlation was observed between measures of tau burden and atrophy in both the hippocampus and cortex (a). Open-field locomotor activity was correlated with all three other behavioural assays: T-maze rewarded alternation, Y-maze spatial novelty preference, and the acquisition of the aversive Y-maze spatial reference memory task (b). Small squares $=4$ months; medium squares $=8$ months; large squares $=12$ months

\section{Discussion}

The present study aimed to extend on the initial phenotypic characterisation of the rTg4510 mouse model and, in doing so, to define a common experimental approach to selection and validation of the most relevant cognitive and behavioural endpoints. Out of the seven behavioural assays tested at a late pathological stage, four were sensitive to tau pathology. Performance in these assays was shown to worsen progressively with age, to correlate well with developing tau pathology, and to be responsive to experimental modulation of tau expression by doxycycline treatment. The confluence of these three characteristics further illustrated that functional measures such as behavioural performance can be a meaningful proxy for underlying pathology, without the need for invasive measures.

The first study assessed 12-month-old male $\operatorname{rTg} 4510$ mice across a range of behavioural tasks to determine which would be sensitive to tau pathology. By this time point, bi-transgenic rTg4510 (CC) mice displayed considerable tau burden and brain atrophy, the extent of which was broadly comparable to that reported previously [18]. CC mice exhibited striking levels of locomotor hyperactivity, profound stereotypy/circling behaviours, and yet intact balance and motor co-ordination. The propensity to become increasingly hyperactive and stereotyped with age is a common characteristic of tau transgenic models [19-21] and needs to be considered when accounting for other behavioural impairments. For instance, male CC mouse performance was no better than chance in both T-maze rewarded alternation and Y-maze novelty preference tasks, though Y-maze continuous alternation performance was just as good as wild-type (WW) mice. Such apparent discrepancy between three tasks of short-term habituation and working memory are likely driven by stereotypy. Circling behaviour may indeed promote or prevent arm alternation respectively in continuous versus discrete-trial alternation paradigms. Several other groups have described behavioural deficits in rTg4510 mice, predominantly in the water maze, but rarely take into consideration how these effects might primarily be driven by stereotypy rather than cognitive dysfunction [21]. Also of interest in this study was that male CC mice could acquire a nonspatial visual cue discrimination maze task as effectively as WW controls. This highlights the ability of the animals to overcome stereotypy in certain scenarios and suggests that some aspects of the $\mathrm{r} \operatorname{Tg} 4510 \mathrm{CC}$ mouse behavioural repertoire are still intact even at this advanced pathological stage. This stands in contrast to previous findings of impairments in visible platform water maze performance in rTg4510 CC mice [16, 22], and suggests that not all tests of visual capacity may offer concordant outcomes. In conclusion, cognitive and behavioural deficits were observed in four out of the seven assays used. Open-field locomotor activity and T-maze alternation measured CC-related deficits of large effect size and lend themselves well to highthroughput longitudinal studies. Aversive Y-maze spatial 


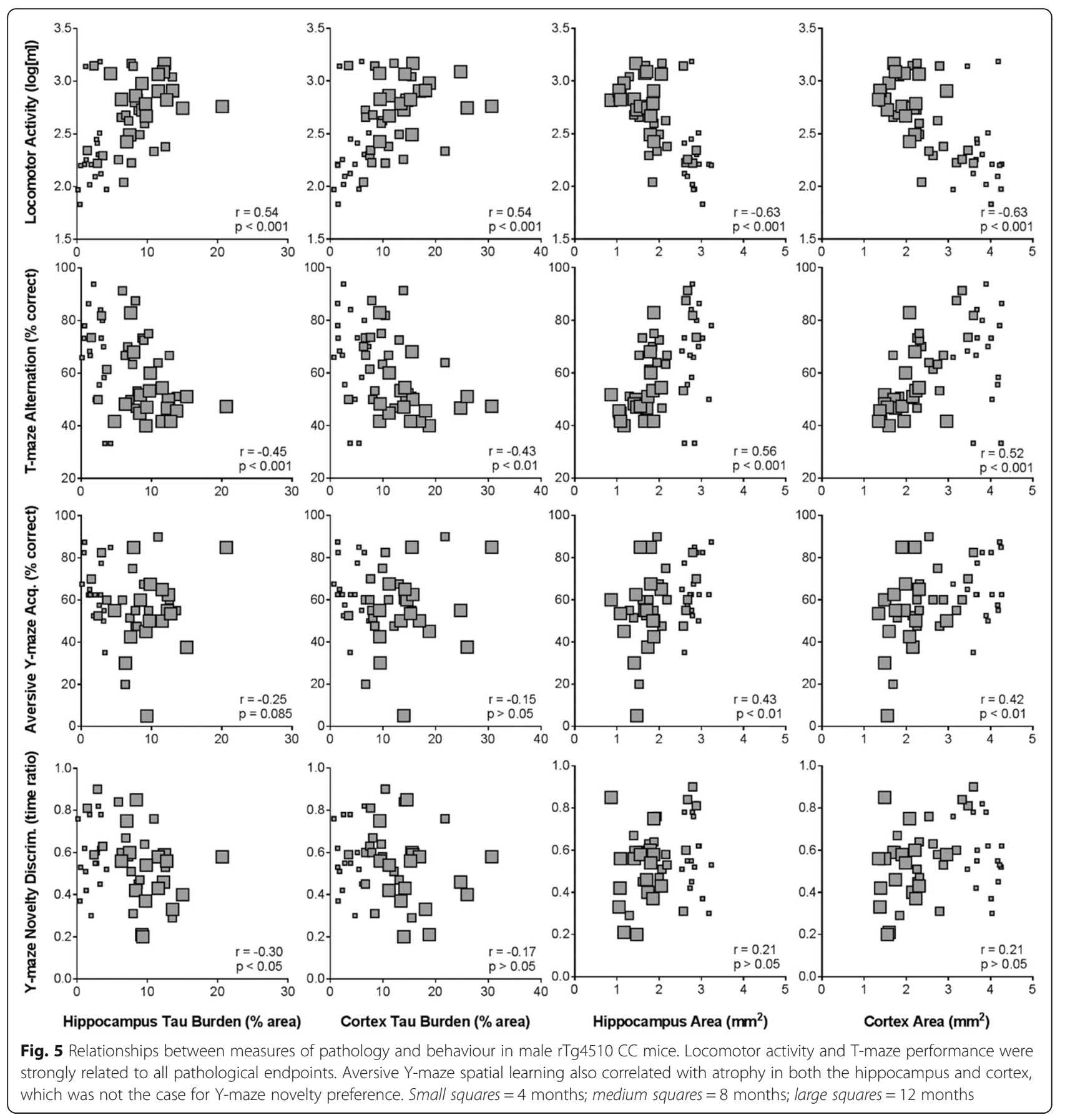

reference memory and Y-maze spatial novelty preference tests were also capable of detecting CC deficits. However, these assays are of lower throughput and may evoke confounding practice effects after repeated testing; these assays are therefore more usefully employed in crosssectional study designs.

In the second study, the four nominated behavioural assays from step 1 were used with the aim of tracking progressive tau-related functional decline from 4 to 12 months of age. Doxycycline was administered from
4.1 months, with the hypothesis that this would prevent further progression of tau pathology, and this in turn would preserve functional capacity. The regional distribution of tau pathology was consistent with that previously observed $[15,23]$, reflecting the use of the calcium/calmodulin-dependent protein kinase II as a promoter for human tau transgene expression in these animals. Tau burden was apparent at 4 months and increased in an age-dependent manner. The progressive increase in tau pathology was associated with brain 
atrophy at 8 and 12 months, believed to be a result of synaptic degradation and neuronal loss [15, 18, 24]. Early research suggested that whilst doxycycline suppression of transgene expression was able to prevent further neuronal loss, tau burden continued to accumulate [18]. The present findings were, however, in line with recent literature [24-27], and suggested that despite only moderate levels of tau transgene suppression (i.e. 50\%) no further tau accumulation and atrophy were observed once doxycycline treatment was initiated. This level of transgene suppression is lower than previously reported by other groups [14]. This is likely due to the food restriction protocol used in this study, where animals would have received less doxycycline than they would have if they were kept on an ad libitum diet and likely were subject to a lower exposure of the drug as a result. Nonetheless, the fact that only a partial suppression of tau expression by doxycycline was able to modulate subsequent progression of tau pathology and normalise behaviour provides encouraging support that potential clinical therapies do not necessarily have to be maximally effective to exhibit functionally beneficial effects.

Locomotor activity and rewarded T-maze alternation performance at 4 months worsened progressively until 12 months, at which point deficits were of similar magnitude to that observed in the first study, providing an internal replication of the dataset. Progressive decline of function was also observed in the aversive Y-maze spatial reference memory task, and was rescued by doxycycline as previously reported elsewhere in the water-maze task $[14,15,21,28]$. Doxycycline treatment effects were bidirectional, preventing both the increase in locomotor activity and the decrease in performance in all three other maze tasks. Such bi-directionality of effects lends weight to the interpretation that doxycycline was truly normalising behaviour. This is an important point, as drug studies often report a restorative effect in only one assay and one direction. Only by combining multiple functional endpoints from tests with different ancillary demands can confidence be raised on the meaningfulness of the observations. Other groups are beginning to take this into consideration when testing therapeutic interventions (e.g. [29]). It is also important to highlight that this work only involved male rTg4510 mice. Sex differences have been reported by other groups [16]; female rTg4510 mice have been shown to develop tau pathology at an earlier age and more aggressively than males. An important next step will be to validate the present findings in female mice.

Correlation analyses revealed that pathological and behavioural endpoints were closely related in CC mice. Locomotor activity and T-maze alternation performance displayed moderate to strong relationships with both measures of pathology, i.e. tau burden and atrophy. Such behavioural measures can therefore be taken as indicative of the level of underlying regional pathology and may offer a longitudinal, non-invasive, and high-throughput in-vivo biomarker of progression (and suppression) of tau pathology. Correlations may ultimately be investigated further with newer pre-clinical imaging techniques such as tau positron emission tomography (PET) tracers $[30,31]$ and multi-parametric magnetic resonance imaging (MRI) [24] that would allow longitudinal assessment of underlying pathology. Such imaging techniques also offer the ability to investigate key features of AD such as atrophy [24], white matter changes [32], and cerebrovascular reactivity [33]. Pairing longitudinal imaging techniques with the behavioural work described here could potentially provide powerful insight into the relationship between functional decline and morphological alterations within diseased brains as the pathology develops.

Given the applied nature of the current work, it is important to comment on how these findings may fit into the bigger picture of AD drug discovery. All pre-clinical models have limitations that should be acknowledged and accounted for wherever possible. Several hundred interventions have been reported to normalise pathological and/or behavioural endpoints in $\mathrm{AD}$ mouse models $[2,11-13]$, with some of these findings reported in $\operatorname{rTg} 4510$ mice [26, 34-36]. It is highly unlikely that any one of the many existing mouse models of AD possesses complete predictive validity. Just as AD patients exhibit various degrees of regional pathological burden and diverse functional alterations [37], AD mouse models also differ in these parameters and may display limited correspondence with human AD canonical Braak staging [38]. Mouse models can also present with pathological and functional artefacts as a consequence of their construction. For instance, the tetracycline transactivator (TTA) used to control tau transgene expression in rTg4510 mice may be linked to developmental and functional abnormalities [39-41]. Since no model is perfect, hypothesis testing in multiple models is always preferable as it offers increased confidence of drug effect via convergent validation [42-44].

\section{Conclusions}

The two-step experimental approach used in this study provides a detailed cognitive and behavioural profiling of male rTg4510 mice, with careful matching to pathological progression. Only four of the seven behavioural assays initially tested produced robust deficits in male rTg4510 mice, highlighting the importance of careful selection and validation of functional endpoints prior to testing. This approach can easily be applied to increase our understanding of other transgenic mouse models of $A D$. Ultimately, better understanding of the nature of functional and pathological decline in such models of 
interest may lead to better study design, and in turn improve confidence in pre-clinical validation of novel, emerging therapeutic interventions. This will hopefully lead to more robust, translatable packages enhancing chances of clinical success in the future.

\section{Abbreviations}

AD: Alzheimer's Disease: ANOVA: Analysis of variance; CC: Bi-transgenic rTg4510 mice; Dox: Doxycycline; IHC: Immunohistochemistry; ITI: Inter-trial interval; RT-qPCR: Reverse transcription quantitative polymerase chain reaction; ROI: Region of interest; rTg4510: rTg(tet-o-TauP301L)4510; WW: Wild-type/non-transgenic rTg4510 mice

\section{Acknowledgements}

We thank Jon Hailwood for technical assistance and David Bannerman for inputs on the development and validation of behavioural assays.

\section{Funding}

All work carried out in this publication was funded by Eli Lilly and Company Limited.

\section{Availability of data and materials}

The datasets used and/or analysed during the current study are available from the corresponding author on reasonable request.

\section{Authors' contributions}

TB performed behavioural testing, analysed and interpreted data, and was the main contributor in writing the manuscript. SM performed immunohistochemical analysis and contributed feedback on the manuscript. TKM performed histology. PJC performed RT-qPCR analysis and contributed feedback on the manuscript. AB contributed to study design and provided feedback on the manuscript. KP contributed feedback on the manuscript and on statistical analysis. BE provided assistance with statistical analysis and contributed feedback on the manuscript. MJO provided feedback on design and on the manuscript. HM provided feedback on the manuscript. ZA contributed to the study design, interpretation of pathological analysis, and writing of the manuscript. GG contributed to study design, interpretation of results, analysis, and writing the manuscript. FG was involved in writing the manuscript, and was a major contributor to study design and analysis, as well as interpreting and analysing data. All authors read and approved the final manuscript.

\section{Ethics approval and consent to participate}

All animal procedures were carried out at Eli Lilly and Company Limited, in accordance with the UK Animals (Scientific Procedures) Act 1986.

\section{Consent for publication}

Not applicable.

\section{Competing interests}

The authors declare being employees of Eli Lilly \& Company Limited at the time this work was completed.

\section{Publisher's Note}

Springer Nature remains neutral with regard to jurisdictional claims in published maps and institutional affiliations.

Received: 12 May 2017 Accepted: 6 September 2017

Published online: 20 September 2017

\section{References}

1. Alzheimer's Association. 2016 Alzheimer's disease facts and figures. Alzheimers Dement. 2016;12(4):459-509.

2. Anand R, Gill KD, Mahdi AA. Therapeutics of Alzheimer's disease: past, present and future. Neuropharmacology. 2014;76(Pt A):27-50.

3. Karran E, De Strooper B. The amyloid cascade hypothesis: are we poised for success or failure? J Neurochem Suppl. 2016;2:237-52.

4. Morgan P, Van Der Graaf PH, Arrowsmith J, Feltner DE, Drummond KS, Wegner CD, Street SD. Can the flow of medicines be improved?
Fundamental pharmacokinetic and pharmacological principles toward improving Phase II survival. Drug Discov Today. 2012;17(9-10):419-24.

5. Soejitno A, Tjan A, Purwata TE. Alzheimer's disease: lessons learned from amyloidocentric clinical trials. CNS Drugs. 2015;29(6):487-502.

6. Drummond E, Wisniewski T. Alzheimer's disease: experimental models and reality. Acta Neuropathol. 2017;133(2):155-75.

7. Busche MA, Grienberger C, Keskin AD, Song B, Neumann U, Staufenbiel M, Forstl $\mathrm{H}$, Konnerth A. Decreased amyloid-beta and increased neuronal hyperactivity by immunotherapy in Alzheimer's models. Nat Neurosci. 2015; 18(12):1725-7.

8. Villain N, Chetelat G, Grassiot B, Bourgeat P, Jones G, Ellis KA, Ames D, Martins RN, Eustache F, Salvado O, Masters CL, Rowe CC, Villemagne VL, AIBL Research Group. Regional dynamics of amyloid-beta deposition in healthy elderly, mild cognitive impairment and Alzheimer's disease: a voxelwise PiB-PET longitudinal study. Brain. 2012;135(Pt 7):2126-39.

9. Brier MR, Gordon B, Friedrichsen K, McCarthy J, Stern A, Christensen J, Owen C, Aldea P, Su Y, Hassenstab J, Cairns NJ, Holtzman DM, Fagan AM, Morris JC, Benzinger TL, Ances BM. Tau and Abeta imaging, CSF measures, and cognition in Alzheimer's disease. Sci Transl Med. 2016;8(338):338ra66.

10. Panza F, Solfrizzi V, Seripa D, Imbimbo BP, Lozupone M, Santamato A, Zecca C, Barulli MR, Bellomo A, Pilotto A, Daniele A, Greco A, Logroscino G. Taucentric targets and drugs in clinical development for the treatment of Alzheimer's disease. Biomed Res Int. 2016;2016:3245935.

11. Luo W, Dou F, Rodina A, Chip S, Kim J, Zhao Q, Moulick K, Aguirre J, Wu N, Greengard P, Chiosis G. Roles of heat-shock protein 90 in maintaining and facilitating the neurodegenerative phenotype in tauopathies. Proc Natl Acad Sci U S A. 2007;104(22):9511-6.

12. Boutajangout A, Quartermain D, Sigurdsson EM. Immunotherapy targeting pathological tau prevents cognitive decline in a new tangle mouse model. J Neurosci. 2010;30(49):16559-66.

13. Troquier L, Caillierez R, Burnouf S, Fernandez-Gomez FJ, Grosjean ME, Zommer N, Sergeant N, Schraen-Maschke S, Blum D, Buee L. Targeting phospho-Ser422 by active Tau immunotherapy in the THYTau22 mouse model: a suitable therapeutic approach. Curr Alzheimer Res. 2012;9(4):397-405.

14. Santacruz K, Lewis J, Spires T, Paulson J, Kotilinek L, Ingelsson M, Guimaraes A, DeTure M, Ramsden M, McGowan E, Forster C, Yue M, Orne J, Janus C, Mariash A, Kuskowski M, Hyman B, Hutton M, Ashe KH. Tau suppression in a neurodegenerative mouse model improves memory function. Science. 2005;309(5733):476-81.

15. Ramsden M, Kotilinek L, Forster C, Paulson J, McGowan E, SantaCruz K, Guimaraes A, Yue M, Lewis J, Carlson G, Hutton M, Ashe KH. Agedependent neurofibrillary tangle formation, neuron loss, and memory impairment in a mouse model of human tauopathy (P301L). J Neurosci. 2005;25(46):10637-47.

16. Yue M, Hanna A, Wilson J, Roder H, Janus C. Sex difference in pathology and memory decline in rTg4510 mouse model of tauopathy. Neurobiol Aging. 2011;32(4):590-603.

17. Ahmed Z, Cooper J, Murray TK, Garn K, McNaughton E, Clarke H, Parhizkar S, Ward MA, Cavallini A, Jackson S, Bose S, Clavaguera F, Tolnay M, Lavenir I, Goedert M, Hutton ML, O'Neill MJ. A novel in vivo model of tau propagation with rapid and progressive neurofibrillary tangle pathology: the pattern of spread is determined by connectivity, not proximity. Acta Neuropathol. 2014;127(5):667-83.

18. Spires TL, Orne JD, SantaCruz K, Pitstick R, Carlson GA, Ashe KH, Hyman BT. Region-specific dissociation of neuronal loss and neurofibrillary pathology in a mouse model of tauopathy. Am J Pathol. 2006;168(5):1598-607.

19. Lewis J, McGowan E, Rockwood J, Melrose H, Nacharaju P, Van Slegtenhorst M, Gwinn-Hardy K, Paul Murphy M, Baker M, Yu X, Duff K, Hardy J, Corral A, Lin WL, Yen SH, Dickson DW, Davies P, Hutton M. Neurofibrillary tangles, amyotrophy and progressive motor disturbance in mice expressing mutant (P301L) tau protein. Nat Genet. 2000;25(4):402-5.

20. Webster SJ, Bachstetter AD, Nelson PT, Schmitt FA, Van Eldik LJ. Using mice to model Alzheimer's dementia: an overview of the clinical disease and the preclinical behavioral changes in 10 mouse models. Front Genet. 2014;5:88.

21. Jul P, Volbracht C, de Jong IE, Helboe L, Elvang AB, Pedersen JT. Hyperactivity with agitative-like behavior in a mouse tauopathy model. J Alzheimers Dis. 2015;49(3):783-95.

22. Bailey RM, Howard J, Knight J, Sahara N, Dickson DW, Lewis J. Effects of the C57BL/6 strain background on tauopathy progression in the rTg4510 mouse model. Mol Neurodegener. 2014;9:8-1326-9-8. 
23. Kopeikina KJ, Polydoro M, Tai HC, Yaeger E, Carlson GA, Pitstick R, Hyman BT, Spires-Jones TL. Synaptic alterations in the rTg4510 mouse model of tauopathy. J Comp Neurol. 2013;521(6):1334-53.

24. Holmes HE, Colgan N, Ismail O, Ma D, Powell NM, O'Callaghan JM, Harrison IF, Johnson RA, Murray TK, Ahmed Z, Heggenes M, Fisher A, Cardoso MJ, Modat M, Walker-Samuel S, Fisher EM, Ourselin S, O'Neill MJ, Wells JA, Collins EC, Lythgoe MF. Imaging the accumulation and suppression of tau pathology using multiparametric MRI. Neurobiol Aging. 2016;39:184-94.

25. Dalby NO, Volbracht C, Helboe L, Larsen PH, Jensen HS, Egebjerg J, Elvang AB. Altered function of hippocampal CA1 pyramidal neurons in the rTg4510 mouse model of tauopathy. J Alzheimers Dis. 2014;40(2):429-42.

26. Radford H, Moreno JA, Verity N, Halliday M, Mallucci GR. PERK inhibition prevents tau-mediated neurodegeneration in a mouse model of frontotemporal dementia. Acta Neuropathol. 2015;130(5):633-42.

27. Blair LJ, Frauen HD, Zhang B, Nordhues BA, Bijan S, Lin YC, Zamudio F, Hernandez LD, Sabbagh JJ, Selenica ML, Dickey CA. Tau depletion prevents progressive blood-brain barrier damage in a mouse model of tauopathy. Acta Neuropathol Commun. 2015;3:8-015-0186-2.

28. Berger Z, Roder H, Hanna A, Carlson A, Rangachari V, Yue M, Wszolek Z, Ashe K, Knight J, Dickson D, Andorfer C, Rosenberry TL, Lewis J, Hutton M, Janus C. Accumulation of pathological tau species and memory loss in a conditional model of tauopathy. J Neurosci. 2007;27(14):3650-62.

29. Selenica ML, Benner L, Housley SB, Manchec B, Lee DC, Nash KR, Kalin J, Bergman JA, Kozikowski A, Gordon MN, Morgan D. Histone deacetylase 6 inhibition improves memory and reduces total tau levels in a mouse model of tau deposition. Alzheimers Res Ther. 2014;6(1):12.

30. Harada R, Okamura N, Furumoto S, Furukawa K, Ishiki A, Tomita N, Tago T, Hiraoka K, Watanuki S, Shidahara M, Miyake M, Ishikawa Y, Matsuda R, Inami A, Yoshikawa T, Funaki Y, Iwata R, Tashiro M, Yanai K, Arai H, Kudo Y. 18 FTHK5351: a novel PET radiotracer for imaging neurofibrillary pathology in Alzheimer disease. J Nucl Med. 2016;57(2):208-14.

31. Smith R, Puschmann A, Scholl M, Ohlsson T, van Swieten J, Honer M, Englund E, Hansson O. 18 F-AV-1451 tau PET imaging correlates strongly with tau neuropathology in MAPT mutation carriers. Brain. 2016;139(Pt 9):2372-9.

32. Sahara N, Perez PD, Lin WL, Dickson DW, Ren Y, Zeng H, Lewis J, Febo M. Age-related decline in white matter integrity in a mouse model of tauopathy: an in vivo diffusion tensor magnetic resonance imaging study. Neurobiol Aging. 2014;35(6):1364-74.

33. Wells JA, Holmes HE, O'Callaghan JM, Colgan N, Ismail O, Fisher EM, Siow B, Murray TK, Schwarz AJ, O'Neill MJ, Collins EC, Lythgoe MF. Increased cerebral vascular reactivity in the tau expressing rTg4510 mouse: evidence against the role of tau pathology to impair vascular health in Alzheimer's disease. J Cereb Blood Flow Metab. 2015:35(3):359-62.

34. Hunt Jr JB, Nash KR, Placides D, Moran P, Selenica ML, Abuqalbeen F, Ratnasamy K, Slouha N, Rodriguez-Ospina S, Savlia M, Ranaweera Y, Reid P, Dickey CA, Uricia R, Yang CG, Sandusky LA, Gordon MN, Morgan D, Lee DC. Sustained arginase 1 expression modulates pathological tau deposits in a mouse model of tauopathy. J Neurosci. 2015;35(44):14842-60.

35. Hunsberger HC, Hickman JE, Reed MN. Riluzole rescues alterations in rapid glutamate transients in the hippocampus of rTg4510 mice. Metab Brain Dis. 2016:31(3):711-5.

36. Schroeder SK, Joly-Amado A, Gordon MN, Morgan D. Tau-directed immunotherapy: a promising strategy for treating Alzheimer's disease and other tauopathies. J Neuroimmune Pharmacol. 2016;11(1):9-25.

37. Schwarz AJ, Yu P, Miller BB, Shcherbinin S, Dickson J, Navitsky M, Joshi AD, Devous MDS, Mintun MS. Regional profiles of the candidate tau PET ligand 18 F-AV-1451 recapitulate key features of Braak histopathological stages. Brain. 2016;139(Pt 5):1539-50.

38. Braak H, Braak E. Staging of Alzheimer's disease-related neurofibrillary changes. Neurobiol Aging. 1995;16(3):271-8. discussion 278-84.

39. Barton MD, Dunlop JW, Psaltis G, Kulik J, DeGennaro L, Kwak SP. Modified GFAP promoter auto-regulates tet-activator expression for increased transactivation and reduced tTA-associated toxicity. Brain Res Mol Brain Res. 2002;101(1-2):71-81.

40. McKinney BC, Schneider JS, Schafer GL, Lowing JL, Mohan S, Zhao MX, Heng MY, Albin RL, Seasholtz AF, Akil H, Murphy GG. Decreased locomotor activity in mice expressing tTA under control of the CaMKII alpha promoter. Genes Brain Behav. 2008;7(2):203-13.

41. Han HJ, Allen CC, Buchovecky CM, Yetman MJ, Born HA, Marin MA, Rodgers SP, Song BJ, Lu HC, Justice MJ, Probst FJ, Jankowsky JL. Strain background influences neurotoxicity and behavioral abnormalities in mice expressing the tetracycline transactivator. J Neurosci. 2012;32(31):10574-86.

42. Shineman DW, Basi GS, Bizon JL, Colton CA, Greenberg BD, Hollister BA, Lincecum J, Leblanc GG, Lee LB, Luo F, Morgan D, Morse I, Refolo LM, Riddell DR, Scearce-Levie K, Sweeney P, Yrjanheikki J, Fillit HM. Accelerating drug discovery for Alzheimer's disease: best practices for preclinical animal studies. Alzheimers Res Ther. 2011;3(5):28.

43. Goetghebeur PJ, Swartz JE. True alignment of preclinical and clinical research to enhance success in CNS drug development: a review of the current evidence. J Psychopharmacol. 2016;30(7):586-94.

44. Luo J, Lee SH, VandeVrede L, Qin Z, Ben Aissa M, Larson J, Teich AF, Arancio O, D'Souza Y, Elharram A, Koster K, Tai LM, LaDu MJ, Bennett BM, Thatcher GR. A multifunctional therapeutic approach to disease modification in multiple familial mouse models and a novel sporadic model of Alzheimer's disease. Mol Neurodegener. 2016;11:35-016-0103-6.

\section{Submit your next manuscript to BioMed Central and we will help you at every step:}

- We accept pre-submission inquiries

- Our selector tool helps you to find the most relevant journal

- We provide round the clock customer support

- Convenient online submission

- Thorough peer review

- Inclusion in PubMed and all major indexing services

- Maximum visibility for your research

Submit your manuscript at www.biomedcentral.com/submit
Ciomed Central 